\title{
Two dimensional simulation of laminar flow by three- jet in a semi-confined space
}

https://doi.org/10.1515/nleng-2020-0001

Received Oct 12, 2019; accepted Oct 23, 2019.

\begin{abstract}
Equipment performance improvement in a wide range of working conditions is one of the major goals of aerodynamics. This goal can be achieved by the deformation of the object being examined or by using flow control techniques in active or inactive modes. In different researches, how to change the development ratio on the semi-confined space with input jet system is surveyed. In this study, two-dimensional simulation of the flow has been investigated in three-jet laminar flow in a semi-confined space. To determine the effective and optimal mixing in a laminar flow, critical Reynolds numbers were determined to distinguish when the flow in the channel from a steady-state symmetric flow formed downstream recirculation and ultimately transient flow. To better understand the flow characteristics, the simulations were changed at a fixed jet spacing (input jets distance to height of space ratio). Also, in this paper, for comparison, four jets were considered. Based on the results, it was observed that in all cases, mixing occurred in the space between three jets. Placing the jet along the walls of the semiconfined space allows the best combination, and increase in the distance between the first and third jets and reduction of the particle coefficient caused to reach the critical Reynolds number faster and, as a result, mixing in a laminar flow with geometric changes of the semi-confined space.
\end{abstract}

Keywords: input jet, laminar, critical Reynolds number, mixing

\section{Introduction}

The mixing process takes place to achieve the same physical properties in the fluid. Different fluids are mixed by mechanical means, such as using mixer (propeller), fluid jets,

\footnotetext{
*Corresponding Author: Mohammad Mosaddeghi, Middle East Technical University Ankara, Çankaya, Turkey,

E-mail: en_mosadeghi@yahoo.com
}

etc. In most cases, an optimal mixing flow can be achieved by natural unstable fluid. In this regard, using symmetric geometric structure is efficient. Several different studies have been conducted on how to change the development ratio affecting the channel input jet system. Tusi \& Wang reported that decreasing the input size would reduce the critical Reynolds number in the system. They not only used tacit solvers to compare implicit simulations, but also could model the non-symmetric stable solving method using explicit solvers [1]. Mishra simulates a simple stepwise transformation asymmetrically using asymmetric grid compared to symmetric gridding [2]. In fact, much research has been done on the volatility of an input from the sudden development of flow. Mizushima and Shiotani, using a stable nonlinear analysis, were able to stage a fairly asymmetric modeling stepwise [3]. These researchers were able to correct asymmetrically the results of Fearn's research work. In the study by Fearn et al., the pitch fork bifurcation diagram is split into two branches. In the elementary branch, there is a simple and uniform transmission of a non-stable quasi-stable solution, and the rest is unstable (9 and 10). By increasing the number of the Reynolds, the flow appears to be three-dimensional before it becomes unstable. This mode is suggested by Fearn et al. [4]. In contrast, for a periodic flow, it remains exclusively two-dimensional $[1,5]$. This is called "Hopf bifurcation".

Jones et al. tested the flows to the water surface and finally presented a table for flow classification and prediction of trajectory and diffusion; it also showed mixing of a two dimensional jet with low Reynolds numbers at a distance specifically, it ends and goes downwards [6]. Jirka analyzes the input jet in shallow waters and states that all of the characteristics related to the concentration and diffusion of jets [7], in the shallow environments, are influenced by the depth of flow which was studied by Cateberson et al. based on sewage from wastewater [8]. They established the output of submerged circular jets, which were discharged horizontally in the receiver's water supply, and investigated the approaching region of the negative density jet. The researchers investigated the inclined jet flow. The results of these experiments showed that the near and distant jet region has a significant effect on each 
other and the analysis of jet diffusion cannot be explained solely on the basis of near jet area. Tang et al. state that the depth and type of foam environment are very important in the distribution and length of the jet path, because the jet flow path at the bottom of the floor, is influenced by the environment of the receiving environment [9]. In a study by Neofytou, a sudden development of symmetry for nonNewtonian fluid was found. The researchers found that similar to the Newtonian flow, the symmetric pattern of breaking occurred at the critical point, which depends on the Reynolds number. In some studies, researchers have also found critical Pinn fork number in pitch fork bifurcation, which can be classified into three categories: bifurcation analysis, numerical simulations and empirical work in the bifurcation laboratory, and stability analysis (Linear and non-linear) $[6,9,10]$ or wide system methods with the exact bifurcation point $[1,11,12]$. For the other two methods, it is necessary to gradually increase or decrease the number of the real number to find the critical point. Also, in these researches, it is difficult to find and determine the critical point in both ways. Researchers such as Foumeny et al. [13] and Schreck [14] abandoned the critical value of the Reynold number close to the theoretical values, but other researchers such as Durst et al. [15] and De Zilwa [16] could not obtain the critical value for the Reynold number in accordance with theoretical results. Sobey and Drazin [17] numerically studied the two dimensional flow in a channel of smooth expansion. By bifurcation theory, their results showed only one steady and symmetrical solution is available at a low Reynolds numbers.

The purpose of this paper is to investigate the conditions governing the problem using 3 input jets of space to maximize the mixing rate in the laminar flow without increasing Reynolds number and turbulence flow. In fact, when the fluid enters the semi-confinedspace through jets, mixing is complete and the flow is somewhat turbulent, but after complete mixing, the flow is slowly established. This work investigated the simulation fluid behavior in a two-dimensional fluctuating environment for triple jets. In this simulation, the flow field and the flow instability in a geometry consisting of triple jets are examined. The main problem is to obtain and divide the flow patterns and Reynolds number according to the onset of instability (changing the flow pattern from time to time dependent on the flow pattern).

\section{Simulation of semi-confined space}

\subsection{Studied geometry}

The geometry (Figure 1) consists of three parallel jets, the center line of one of the jets is always in the middle of the channel and the distance between the center lines of the two jets is high and low equaling to $\mathrm{S}$.

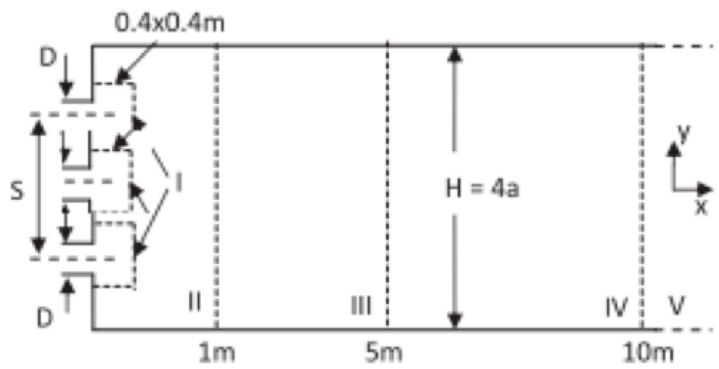

Figure 1: The two dimensional solution domain with three jets in a confined space

The main problem in this study is the effect of three dimensional by non-dimensional numbers on the flow pattern:

- Reynolds number: In this research, the maximum Reynolds number is 35 .

The governing equations are as follows:

$$
\begin{gathered}
\nabla \cdot v=0 \\
\frac{\partial v}{\partial \tau}+(v \cdot \nabla) v=-\nabla p+R e^{-1} \nabla^{2} v \\
R e=\frac{\rho U D_{a v e}}{\mu} \\
\frac{\partial \phi}{\partial \tau}+\mu_{c} \frac{\partial \phi}{\partial x}+v_{c} \frac{\partial \phi}{\partial y}=0
\end{gathered}
$$

where Re, represents the jet Reynolds number, $v$, fluid kinematic viscosity, $\mathrm{p}$, pressure, $\mathrm{u}_{c}$ and $v_{c}$ are convective velocity components.

- The distance between two jets to channel Height ratio (S/H)

- Aspect Ratio channel $(y=3 \mathrm{D} / \mathrm{H})$

To reach this goal, the ratio of $\mathrm{S} / \mathrm{H}$ to the values of 0.25 , $0.5,0.75$, and 0.9 was modified by first fixing the aspect ratio $(y)$, and the effect of increasing the Reynolds number 
was studied. Also, in order to study effect of the paradigm, given $(\mathrm{S} / \mathrm{H}=0.5)$, the values of $0.15,0.1$ and 0.05 for the aspect ratio were studied. In each Reynolds case, the transition from a stable flow to a time-dependent flow was solved for Reynolds number above this time-dependent flow, and the results were examined in the final step of the experiment.

It should be noted that due to the limited time and system resources, only 60 seconds of simulated flow (time step of 0.1 seconds, 600 steps) are simulated. In this case, the time to solve some of the modes has reached more than 12 hours. It is advisable to continue, if possible, for at least 3 minutes.

\subsection{Drawing geometry in design modeler environment}

Geometry is first drawn up in the environment. For example, the geometry for $\mathrm{S} / \mathrm{H}=0.25, y=0.15$ and $\mathrm{D}=0.2 \mathrm{~m}$ is as follows in Figure 2:

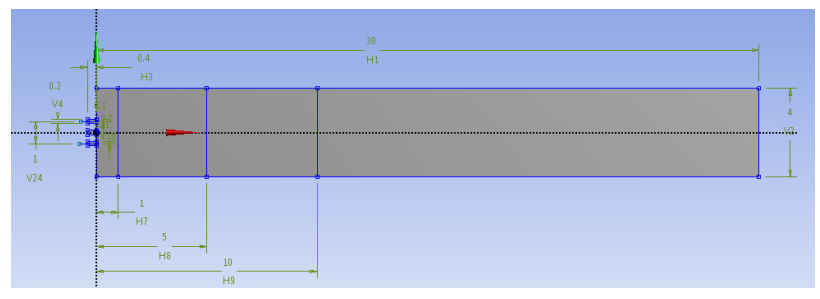

Figure 2: The geometry of confined space: $\mathrm{S} / \mathrm{H}=0.25, y=0.15$ and $\mathrm{D}=0.2 \mathrm{~m}$

It is designed for each geometry separately in the ANSYS Meshing environment. The maximum mesh size is $0.05 \mathrm{~m}$. The rectangular grid was used for calculations and the density of the grid was carefully selected to ensure the physical accuracy of the flow (Figure 3).

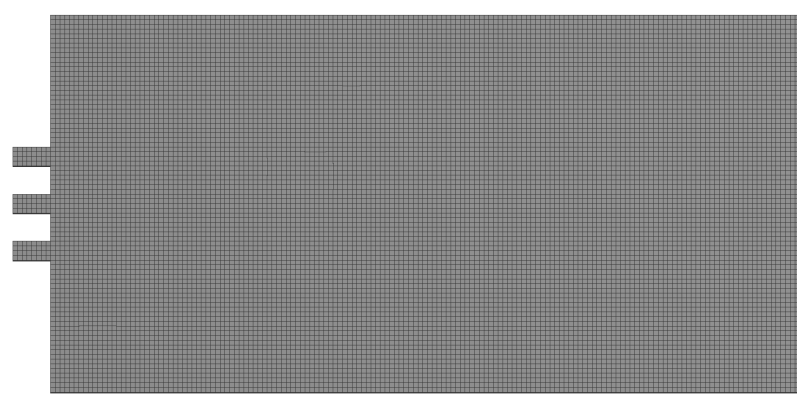

Figure 3: Meshing environment
The border areas were designated to apply boundary conditions as shown in Figure 4.
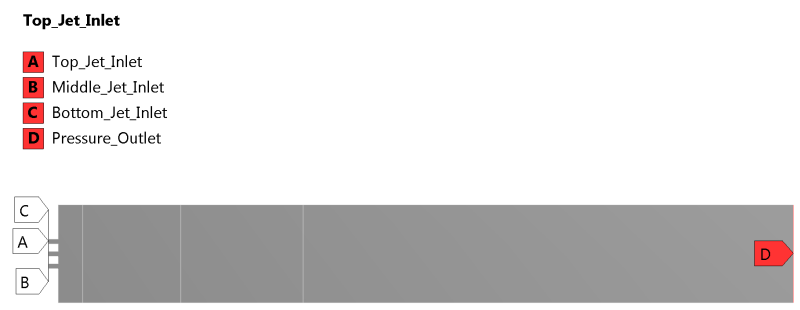

Figure 4: The boundary conditions for confined space

Non-slip boundary conditions are considered on the confining walls. At the three jet-inlets of channel a uniform distribution of velocity is determined.

It is known that for a completely separate flow completely developed with a cross section, the maximum speed of flow is twice as fast as the compressed speed, while the two-dimensional channel flow rate is 1.5. Hence, the flow with relatively low proportions requires longer input lengths than those with large proportions than fullscale development. With Running Fluent, results are reviewed and stored in the CFD-Post environment. We noted that properties were defined for fluid as flowed: $\rho=1, \mu=\mathrm{D}$

$$
R e=U \_a v e
$$

As a result, the input speed determines the Reynolds number of the fluid, and only this boundary condition will change between different modes.

\section{Results}

\subsection{The effect of $\mathrm{S} / \mathrm{H}$ on the flow pattern}

By transferring jets to channel edges, they often have an asymmetric flow, and this is similar to that of a jet. As the Reynold number increases, the jets may become unstable. It has been determined that the interaction of jet with jet on bifurcation has a much stronger wall effect. Tzeng (1998) said that an increase in the distance between the two jets or increase in the Reynolds number moved the jet flow to a point where a strong centralized current was created. In various studies, the effect of the symmetric geometry shape on unstable behavior in symmetric flows was investigated. This point is of such as great importance that volatility occurs in the course of how and when. In this case, the design of optimal models is easy and the mixing 
can be increased without increasing the Reynolds number, using change of structural parameters.

It can be seen from Figure 5 that with an increase in the $\mathrm{S} / \mathrm{H}$ value, the critical Reynolds number will be reached faster in the studied state. it means that by increasing the $\mathrm{S} / \mathrm{H}$ ratio, the flow of the inlet fluid from the jets will go out uniformly earlier and will become unstable. Instability is less important in Reynolds, because it shows that as $\mathrm{S} / \mathrm{H}$ increases, the flow is quite laminar and in the low Reynolds number, instability occurs and the mixing occurs through three input jets. Therefore, based on shape of the flow characteristics, the geometric shape and the distance between the jets $(\mathrm{S} / \mathrm{H})$ have a great influence on the flow behavior within the semi-confined space. Given the flow behavior in the given form, it can be seen that the stable state is asymmetric.

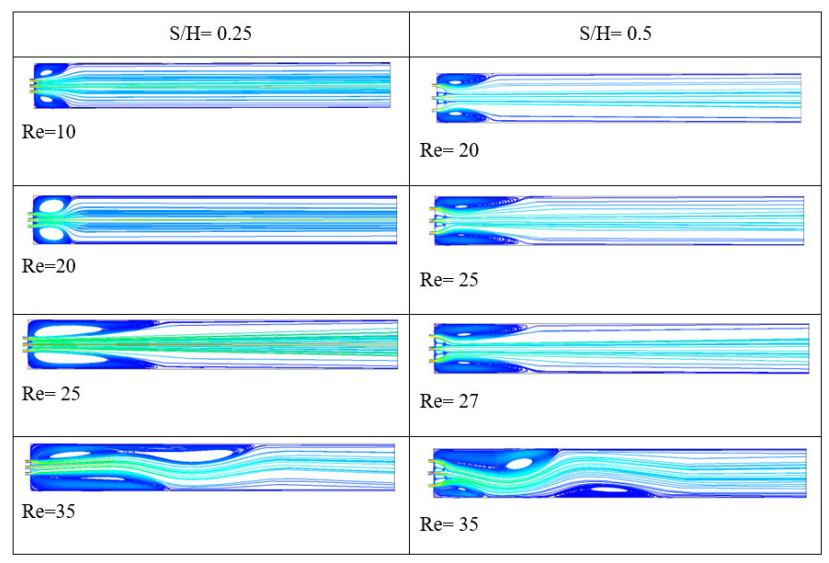

Figure 5: Streamlines of characteristic flows for $\mathrm{S} / \mathrm{H}=0.25,0.5$ in three jets

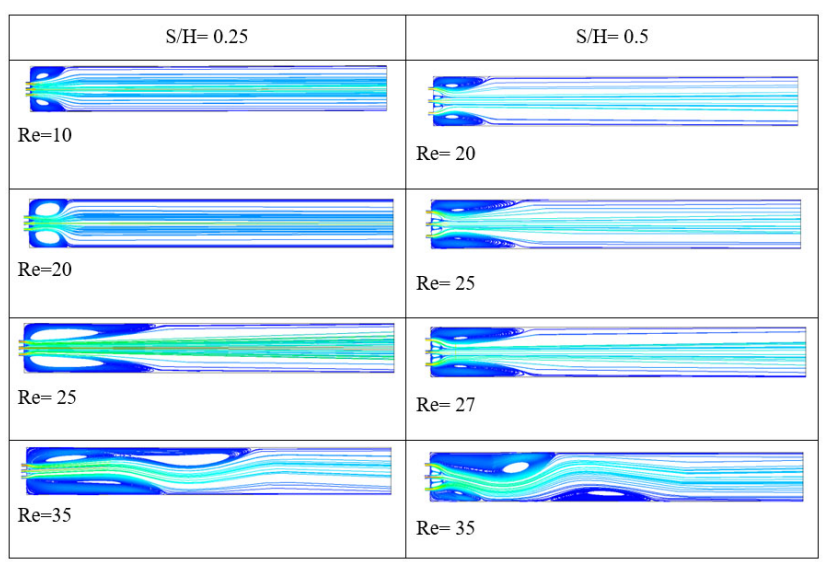

Figure 6: Streamlines of characteristic flows for $\mathrm{S} / \mathrm{H}=0.75,0.9$ in three jets
In steady asymmetric inward flow, it is seen that the flow of the jets from each jet is reinforced by other jets because of parallel input jets, and at the same initial moments and near the entrance, the three input streams are completely mixed together. Indeed, this mode changes the effect of the Coanda effect, and all three input flows from the walls are combined, and the flow in the channel center is amplified and flow behavior proceeds along the path such as the flow of the jet [12]. It is very important that using three jets of input flow at the initial moments faster than the similar scenarios for the two jets, and the Reynolds obtained for different $\mathrm{S} / \mathrm{H}$ states indicate that no matter how much $\mathrm{S} / \mathrm{H}$ that is, the distance between the two jets is low, the flow will be steady and will be more unstable in the higher Reynolds number. For example, it can be seen in the Figure 6 that in $\mathrm{S} / \mathrm{H}=0.5$, the critical Reynold is 35. However, for $\mathrm{S} / \mathrm{H}=0.9$, although the critical Reynolds number is 35, in lower Reynolds number, such as $R e=16$, it is also a non-destructive state in the flow. Therefore, the mixing process is less likely to occur in the lower Reynolds, increasing the distance between the two upper and lower jets from one another and moving them near the upper and lower walls in the channel (Figure 6).

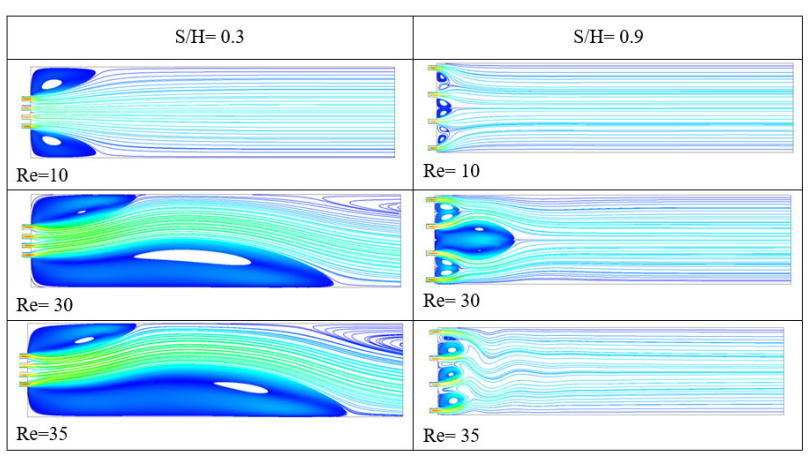

Figure 7: Streamlines of characteristic flows for $\mathrm{S} / \mathrm{H}=0.3,0.9$ in four jets

Comparison of Figure 5 with 7 shows that there is no significant difference in the mixing rate for three jet with four jet in a channel with the same dimensions.

Due to changes in Reynolds number, the unsteady start with $\mathrm{S} / \mathrm{H}$ is approximately shown in Table 1.

An interesting point about the critical Reynolds number needs to be addressed in the following. It can be understood that before this critical point has been reached, a slight deviation between the two reattachment lengths has been progressing. A similar phenomenon was also taken into account in non-Newtonian flow calculations by Neofytou and Drikakis [18] and can be identified in 
Table 1: Critical Reynolds number in different $\mathrm{S} / \mathrm{H}$ for three jets

\begin{tabular}{ccc}
\hline $\mathbf{S} / \mathbf{H}$ & $\mathbf{R e}$ & $\mathbf{R e}_{c r}$ \\
\hline 0.25 & $10,20,25,35$ & 35 \\
0.5 & $20,25,26.8,27,35$ & $\mathbf{2 7 , 3 5}$ \\
0.75 & $10,18,20,35$ & $\mathbf{2 0 , 3 5}$ \\
0.9 & $10,16,20,35$ & $\mathbf{1 6 , 2 0 , 3 5}$ \\
\hline
\end{tabular}

two-dimensional diagrams in Schreck and Schafer's threedimensional study [14].

It is also observed that changes in the Reynolds number have less steady flow to the walls, and concentric circles are created along the flow sides, which is enhanced by increasing Reynolds number and circles are drawn from the sides to the center of the semi-confined space and the turbulent state is observed in the flow. In fact, in lower $\mathrm{S} / \mathrm{H}$, there is more symmetric mode at the input jet, and there is Eddy mode between each jet. Another point is that an increase in the $\mathrm{S} / \mathrm{H}$ reduces the size of the vortices created in the walls and, in fact, the flow goes back to a laminar and stable state. This is completely evident in the vortices generated in the flow path for $\mathrm{S} / \mathrm{H}=0.75,0.9$.

In this paper, to investigate fluid velocity profile, velocity vector contours are used for three jets and four jets that were showed in Figures 8 and 9.

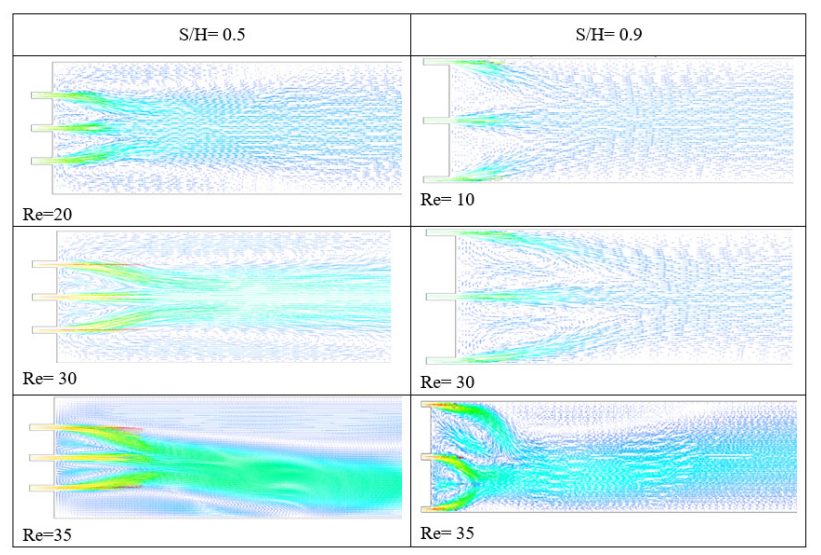

Figure 8: Velocity vector of characteristic flows for $\mathrm{S} / \mathrm{H}=0.5,0.9$ in three jets

According to the velocity vectors in Figure 8 , it can be seen that in both cases examined by increasing Re, mixing in the fluid increases.

According to the velocity vector, using four jets, it can be seen that in the lower $\mathrm{S} / \mathrm{H}$, by increasing Re, increases mixing in the fluid. According to the Figure 9, it can be seen that in $\mathrm{S} / \mathrm{H}=0.9$, with the increase of Re, the mixing of the fluid occurs at the beginning of the channel length.

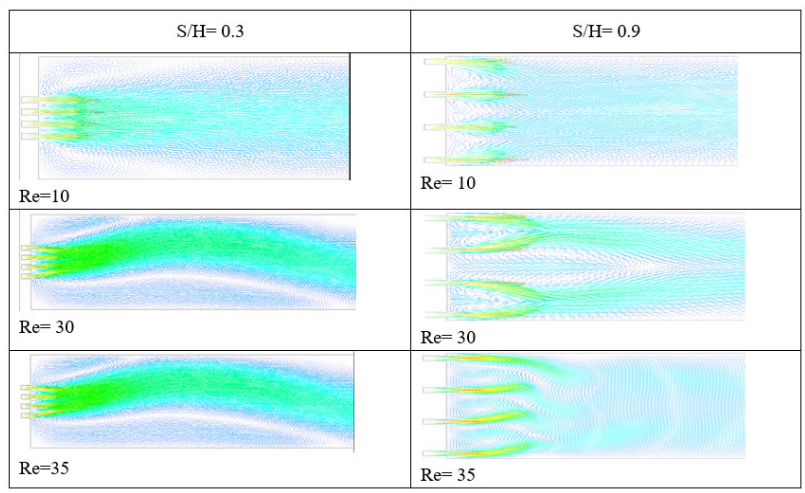

Figure 9: Velocity vector of characteristic flows for $\mathrm{S} / \mathrm{H}=0.3,0.9$ in four jets

\subsection{The effect of $y$ on the flow pattern}

In this section, with increasing $\mathrm{H}$, the channel height of the Aspect Ratio decreases and its effect on the flow is investigated.

According to Figure ??, it can be seen that due to increase in the values of Reynolds number to the critical Reynolds, the instability of the flow considerably increases, so that in the downstream of the Reynolds number, the flow reaches instability and higher gains leading to the occurrence of faster instability. In fact, with the increase of $y$, the critical number of Reynolds decreases and the flow rapidly becomes unstable.

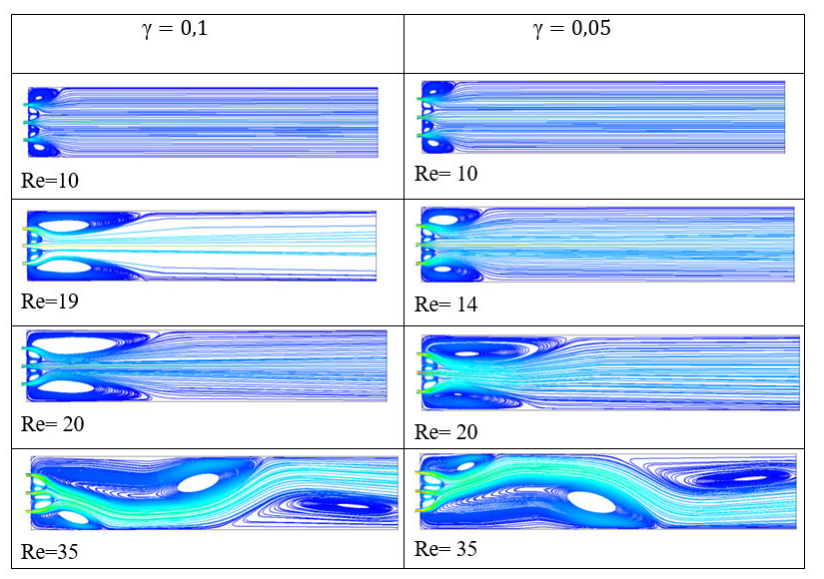

Figure 10: Streamlines of characteristic flows for $y=0.05,0.1$ in three jets

In the span wise direction and near the entrance, we can see the three vortices with different size. In the region near the side wall and in up and down sections, there are two large vortexes that these vortexes expand their sizes in the horizontal direction. In many researches, limiting 
streamlines (or skin friction lines) have been widely used to investigate three-dimensional separated flows. In topology theory singular points occur in places where skin friction is zero. Singular points are classified into nodes and saddles. The nodes can be divided into base/ nodal points and focal points.

In Figure 10, At $y=0.1$, with increasing Re, the streamlines on the side wall show that either the large vortex near the upper step or the third vortex on the lower wall is converted to the focal point of the attachment, which acts as a source of limiting streamlines that draws fluid from the core region and penetrates of the wall surface. The small vortex below the lower stage turns into a focal point of separation which behaves like a sink in which the liquid collected from the wall surface eventually disappears. This indicates that a particle that is initially near this point is transmitted to the channel core. Separation points and reconnection of the third vortex in the bottom wall are converted to saddle points.

points occur where skin friction. Evolutionary points are classified into nodes and saddles.

The start of instability with the particle coefficient $(y)$ is as follows $(\mathrm{S} / \mathrm{H}=0.5)$ in Table 2.

Table 2: Critical Reynolds number in different $y$ for three jets

\begin{tabular}{ccc}
\hline $\boldsymbol{y}$ & $\mathbf{R e}$ & $\mathbf{R e}_{c r}$ \\
\hline 0.15 & $10,19,20,35$ & $\mathbf{2 0 , 3 5}$ \\
0.1 & $10,14,20,35$ & $\mathbf{2 0 , 3 5}$ \\
0.5 & $20,25,26.8,27,35$ & $\mathbf{2 7 , 3 5}$ \\
\hline
\end{tabular}

\subsection{The effect of pressure on the flow pattern}

The pattern of pressure variations in the system is as shown in Figure 11.

According to Figure 11, it can be seen that with the increase of the values of the Reynolds numbers, at $y=0.1$ and $y=0.05$, the pressure on the system increases, at $\operatorname{Re}=35$, the pressure in the three jets are intense and with increasing pressure in the system, turbulence in the intra channel flow is observed. It seems to be in $y=0.05$ than $y=0.1$ it is higher pressure.

If four jets are used (Figure 12), it can be seen that increasing the distance between the jets, the fluid pressure is higher at the inlet and the mixing is more intense in this region. As the length of channel increases, the pressure and the mixing rate decreases.

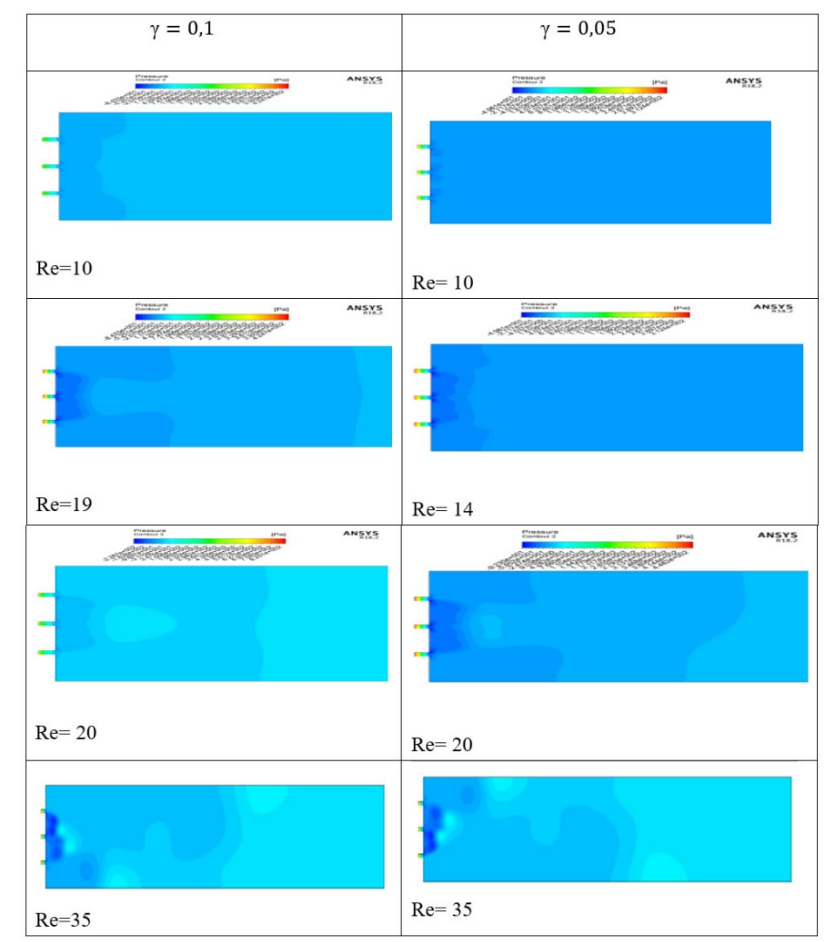

Figure 11: The pressure of flow for $y=0.05,0.1$ in different Reynolds numbers for three jets

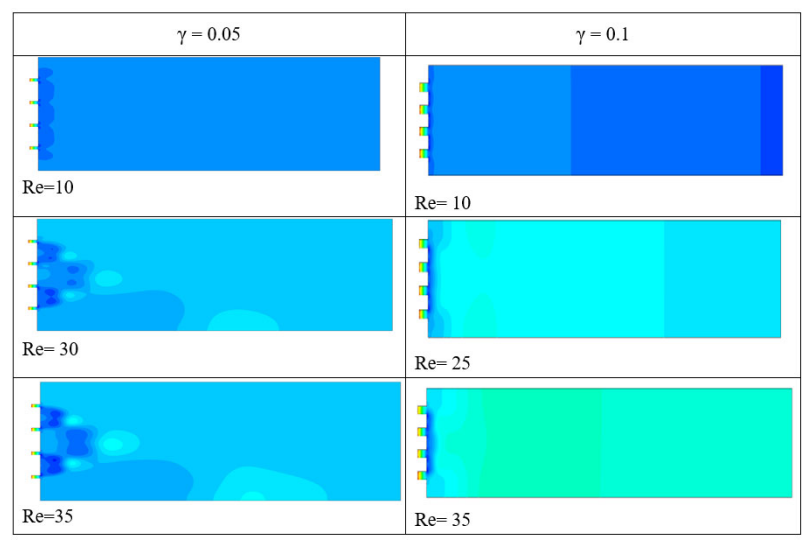

Figure 12: The pressure of flow for $y=0.05,0.1$ in different Reynolds numbers for four jets

\subsection{The effect of channel length on the flow}

In the Figure 13, streamlines of characteristic flows and fluid velocity have been displayed.

It is observed from Figure 13 that by increasing in the channel length, reduces the size of the vortices created in the walls and, in fact, the flow goes back to a laminar and stable state. The velocity of fluid along of the channel length decreases and the flow becomes uniform. 


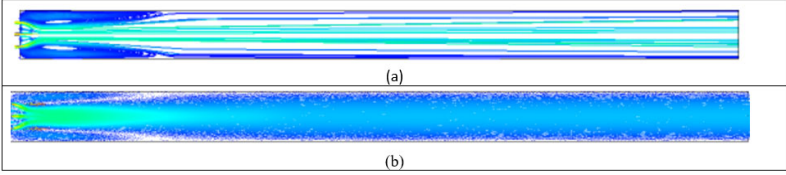

Figure 13: The effect of channel length on the flow for three jets in $\mathrm{Re}=35 \mathrm{a}$ ) streamlines, $\mathrm{b}$ ) velocity of fluid

\section{Conclusion}

Three jets were studied in two dimensions in limited spaces under different geometries, input properties and jet distance. In this study, for comparison, four jets were also considered. The results showed that the lack of current measurements yields good correlation about the development factors. There are three different patterns in the fluid field:

1. Stable and different (lower Reynolds numbers)

2. Unstable and symmetric (medium Reynolds numbers)

3. Unstable and asymmetric (high Reynolds numbers)

As a result, the critical Reynolds number decreases with increasing $\mathrm{S} / \mathrm{H}$ and reducing the Aspect Ratio, $y$.

It has been observed that the use of four jets in comparison to three jets does not have much effect on the flow characteristics inside the channel. Therefore, it is preferred to use three jets. also, along of the channel length, the size of created vortices in the near walls was been decreased and the laminar flow through a sudden expansion in a channel would be separated.

\section{References}

[1] Tsui Y.-Y., Wang H.-W., Side-wall effects on the birfucation of the flow through a sudden expansion. Int. J. Numer. Methods Fluids, 2008, 56(2), 167-184.

[2] Mishra S., Jayaraman K., Asymmetric flows in planar symmetric channels with large expansion ratio. Int. J. Numer. Methods Fluids, 2002, 38(10), 945-962.

[3] Mizushima J., Shiotani Y., Structural instability of the bifurcation diagram for two-dimensional flow in a channel with a sudden expansion, S J. Fluid Mech, 2000, 420(1), 131-145.

[4] Fearn R.M., Cliffe K.A., Nonlinear flow phenomena in a symmetric sudden expansion, J. Fluid Mech., 1990, 211, 595-608.

[5] Revuelta A., On the two-dimensional flow in a sudden expansion with large expansion ratios, Phys. Fluids, 2005, 17(2), 028102.

[6] Jones G., Nash J., Doneker R., Jirka H.G., Buoyant surface discharges into water bodies. I: Flow classification and prediction methodology, J. Hydraulic Eng., ASCE., 2007, 133(9), 10101020.
[7] Jirka H.G., Life study of plane jet bounded in shallow water layer, J. Hydraulic Eng., ASCE, 1999, 125(8), 817-826.

[8] Cuthberston A.J..S., Davies P., Deposition from particle-laden. round, turbulent, horizontal buoyant jets in stationary and following receiving fluids, J. Hydraulic Eng., ASCE, 2008, 134(4), 390-402.

[9] Tang H., Paik J., Sotiropoulos F., Khangaonkar T., ThreeDimensional numerical modeling of initial mixing of thermal discharges at real-life configurations, J. Hydraulic Eng., ASCE, 2008, 134(49), 1210-1224.

[10] Wright S.J., Effects of ambient cross flow and density at ratification on the characteristic behavior of round turbulent buoyant jets, Report No. KH-R-36, W.M KECK Lab. of Hydraulic and Water Resort, 1977, California Inset.

[11] Islam M.R., Zhu D.Z., Flow upstream of two-dimensional intakes. J. Hydraulic Eng., ASCE., 2011, 137(1), 129-134.

[12] Rafferty I., Kaminski D., Non-dimensionalization and mixing quantification of laminar twin semi-confined jets, Int. J. Heat Fluid Flow, 2014, 48, 15-23.

[13] Foumeny E.A., Walker A.J., Bifurcations of incompressible flow through plane symmetric channel expansions, Comput. Fluids, 1996, 25, 335-351.

[14] Schreck E., Schäfer M., Numerical study of bifurcation in threedimensional sudden channel expansions, Comput. Fluids, 2000, 29, 583-593.

[15] Durst F., Tropea C., The plane symmetric sudden-expansion flow at low Reynolds numbers. Journal of Fluid Mechanics, , 1993. 248: p. 567-581.

[16] De Zilwa S.R.N., Whitelaw J.H., Flows through plane suddenexpansions, Int. J. Numer. Meth. Fluids, 2000, 32, 313-329.

[17] Sobey I.J., Drazin P.G., Bifurcation of two-dimensional channel flow, J. Fluid Mech., 1986, 171, 263.

[18] Neofytou P., Drikakis D., Non-Newtonian flow instability in a channel with a sudden expansion, J. Non-Newtonian Fluid Mech., 2003, 111, 127-150. 